\title{
Age-Related Features in Systemic Inflammatory Response in Male Wistar Rats with Different Hypoxia Tolerance ${ }^{\dagger}$
}

\author{
Dzhuliia Dzhalilova ${ }^{1, *(\mathbb{D})}$, Anna Kosyreva ${ }^{1,2}$, Polina Vishnyakova ${ }^{2,3}$, Ivan Tsvetkov ${ }^{1}{ }^{1}$, Natalia Zolotova ${ }^{1}$, \\ Vladimir Mkhitarov ${ }^{1}$ and Olga Makarova ${ }^{1}$
}

1 Federal State Budgetary Institution “A.P. Avtsyn Research Institute of Human Morphology", Moscow 117418, Russia; babushka84@list.ru (A.K.); davedm66@gmail.com (I.T.); natashazltv@gmail.com (N.Z.); mkhitarov@mail.ru (V.M.); makarov.olga2013@yandex.ru (O.M.)

2 Histology Department, peoples Friendship University of Russia (RUDN University), Moscow 117198, Russia; vishnyakovapolina@gmail.com

3 National Medical Research Center for Obstetrics, Gynecology and Perinatology Named after Academician V.I. Kulakov of Ministry of Healthcare of Russian Federation, Moscow 117997, Russia

* Correspondence: juliaja193@mail.ru

+ Presented at the 1st International Electronic Conference on Molecular Sciences: Druggable Targets of Emerging Infectious Diseases (ECMS 2021), 1-14 September 2021; Available online: https:/ / ecms2021.sciforum.net/.

Citation: Dzhalilova, D.; Kosyreva A.; Vishnyakova, P.; Tsvetkov, I.; Zolotova, N.; Mkhitarov, V.; Makarova, O. Age-Related Features in Systemic Inflammatory Response in Male Wistar Rats with Different Hypoxia Tolerance. Med. Sci. Forum 2022, 7, 4. https://doi.org/10.3390/ ECMS2021-10831

Academic Editor: Claudiu

T. Supuran

Published: 26 January 2022

Publisher's Note: MDPI stays neutral with regard to jurisdictional claims in published maps and institutional affiliations.

Copyright: (C) 2022 by the authors. Licensee MDPI, Basel, Switzerland. This article is an open access article distributed under the terms and conditions of the Creative Commons Attribution (CC BY) license (https:// creativecommons.org/licenses/by/ $4.0 /)$.

\begin{abstract}
An organism's hypoxia tolerance depends on many factors, including age. High newborn organism tolerance and high levels of oxidative stress throughout aging have been demonstrated by many studies. However, there is still lack of investigations reflecting the intensity of systemic inflammatory response in organisms of different ages in correlation to hypoxia tolerance. The aim of this study was to determine the relationship between age-related tolerance to hypoxia, HIF-1 and PHD2 (prolyl hydroxylase domain protein) expression levels and the intensity of systemic inflammatory response in newborn, prepubertal and adult Wistar rats. In case of investigation of the tolerance to hypoxia, rats were placed into a decompression chamber at altitude simulation of 11,500 $\mathrm{m}$. It was demonstrated that prepubertal rats are the least tolerant to hypoxia and newborns are the most tolerant. Newborn rats are characterized by high mRNA Hif- $1 \alpha$ expression level in the liver, accompanied by low content of HIF-1 protein and a high level of PHD2. The growth in HIF-1 $\alpha$ protein level with age is accompanied by growth in the level of proinflammatory cytokines. Prepubertal animals are the least hypoxia tolerant, and their HIF- $1 \alpha$ mRNA expression level was higher than in adult animals. The PHD2 activity in prepubertal animals was significantly reduced in comparison to newborn rats, and the HIF-1 $\alpha$ protein level was not changed. The lowest tolerance of the prepubertal males to hypoxia correlated with the greatest manifestations of hepatic inflammation and elevated endotoxin, neopterin, and C-reactive protein levels in LPS-induced systemic inflammatory response. The growth in serum HIF- $1 \alpha 3 \mathrm{~h}$ after LPS injection was observed only in prepubertal rats. The obtained data should be taken into account during the development of a therapeutic strategy for prepubertal children with infectious and inflammatory diseases. Hopefully, this study will provide new insights into age-related differences in tolerance to hypoxia. The possible perspectives of this investigation could be understanding the aspects of HIF-1 mRNA and protein expression in aged animals. Moreover, further studies are required for the identification of additional mechanisms to determine HIF- $1 \alpha$ protein level regulation in prepubertal animals.
\end{abstract}

Keywords: hypoxia tolerance; HIF-1; PHD2; age-related differences; systemic inflammatory response; prepubertal age 
Supplementary Materials: The following supporting information can be downloaded at: https: / / www.mdpi.com/article/10.3390/ECMS2021-10831/s1.

Author Contributions: Conceptualization, D.D. and A.K.; methodology, P.V. and I.T.; software, V.M.; validation, N.Z.; writing — original draft preparation, D.D. and A.K.; writing-review and editing, O.M. All authors discussed the results and commented on the manuscript. All authors have read and agreed to the published version of the manuscript.

Funding: This research was funded by the Ministry of Science and Higher Education of the Russian Federation (No. AAAA-A19-119021490067-4) and by the RUDN University Strategic Academic Leadership Program.

Institutional Review Board Statement: The animal study protocol was approved by Bioethics Committee of the Federal State Budgetary Institution “A.P. Avtsyn Research Institute of Human Morphology" (Protocol No. 21, 29 March 2019).

Informed Consent Statement: Not applicable.

Data Availability Statement: The data presented in this study are available in Supplementary Material.

Conflicts of Interest: The authors declare no conflict of interest. 